\title{
ASpin-NMR data reporting tool
}

\author{
Francesco Sinopoli ${ }^{1}$ and Alessandro Sinopoli ${ }^{1, *}$ \\ 1 Qatar Environment \& Energy Institute (QEERI), Hamad Bin Khalifa University (HBKU), Doha. \\ * Correspondence: asinopoli@hbku.edu.qa
}

Received: 10 August 2019; Accepted: 17 September 2019; Published: 2 November 2019.

\begin{abstract}
Nuclear Magnetic Resonance spectroscopy, commonly referred as NMR spectroscopy, is a widely used technique for determining the structure of organic compounds and metallorganic complexes. The NMR characterization process can be done automatically by specific tools integrated into the main NMR dedicated software, however it is commonly subjected to user's interpretation therefore it still remains a manual process. In relationship with the number of signals to interpret and the complexity of the spectrum, the user usually spends between ten minutes and one hour to manually generate an NMR report, which can be clearly affected by typos or format mismatches. Here we report an Excel sheet-based NMR data reporting tool which, thanks to ad hoc macros, generates as output a formatted NMR report, ready to be directly pasted into any manuscript. ASpin tool leaves to the users the full possibility to interpret the spectrum in this way they can gain more experience in the interpretation process, without giving up the time saving.
\end{abstract}

Keywords: Ferrous metals, crude oils, corrosion rate, corrosive properties, hardness.

\section{Background}

U uclear Magnetic Resonance spectroscopy, commonly referred as NMR spectroscopy, is a widely used 1 technique for determining the structure of organic compounds and metallorganic complexes. Without going into details, when placed in a magnetic field, NMR active nuclei (nuclei of atoms that possess both magnetic moment and angular momentum, such as $1 \mathrm{H}$ or 13C) absorb electromagnetic radiation at a frequency characteristic of each isotope. The resonant frequency, adsorbed energy, and the intensity of the signal are proportional to the strength of an external magnetic field. The magnetic environment around each atom of a molecule changes the resonant frequency, giving access to details of the electronic structure of a compound and its distinctive functional groups. Unlike infrared and UV-visible spectroscopy, where the position of absorption peaks is described by a frequency or wavelength, the position of different NMR resonance signals is dependent on both the external magnetic field strength and the reference frequency, hence a complete analysis and interpretation of the entire spectrum is normally expected when characterizing new synthetic molecules or to verify the purity of a known one.

NMR interpretation plays an essential role in molecular identifications. The structure of an unknown compound, as well as for known structures, can be solved by interpreting NMR spectra through identification of several factors such as chemical shift, spin multiplicity, coupling constants, and integration. All these information can be only extracted after a deep analysis of the NMR spectrum, in particular peak integration and chemical shift are functions commonly integrated into NMR dedicated software (e.g. TopSpin by Bruker, VnmrJ by Agilent, MestReNova by Mestrelab Research); the coupling constants are strictly related with the multiplicity and although they can be calculated by some tools within the main dedicated software, for spectra with not well separated or not well resolved peaks their interpretation is subjected to the user judgement and experience. Reporting the NMR characterization of a molecule is a fundamental requirement for any thesis, manuscript, assignment and report, in the field of synthetic chemistry. For this reason, all the main chemistry journals added in their guidelines for authors, specific formats for reporting the NMR data. A general $1 \mathrm{H}$ NMR format includes: the analyzed nucleus, the used deuterated solvent and the chemical shift $(\delta)$ of each peak from the reference in parts per million (ppm); for each peak the report states the number of corresponding hydrogens; the multiplicity indicated as singlet(s), doublet(d), triplet(t), quartet(q), etc; the coupling constant for each peak in Hertz $(\mathrm{Hz})$. 


\section{The concept}

The NMR characterization process can be done automatically by specific add-ons (such as Bruker CMC and ACD/NMR Workbook Suite) integrated into the main NMR dedicated software, however it commonly remains subjected to user's interpretation therefore it is traditionally a manual process. Also, the use of such automated software for NMR interpretation is generally discouraged by academics, indeed the ability of interpreting and understanding the NMR spectra requires a lot of practice and experience. In relationship with the number of signals to interpret, and the complexity of the spectrum, the user usually spends between 10 minutes and one hour to manually generate an NMR report, which can be clearly affected by typos or format mismatches. Here we present "ASpin", an Excel sheet-based NMR data reporting tool which includes specific macros able to generate as output a formatted NMR report, ready to be directly pasted into any manuscript. Any NMR dedicated software can generate a XY-like output indicating the peak shifts inside a selected region of NMR spectrum; the chemical shift column can be then pasted into ASpin Microsoft Excel interface. At this point the user has to colour the cells containing the shift values accordingly with the assigned multiplicity, in few clicks ASpin will generate the desired NMR report. The advantages in using the ASpin tool are multiple, the most obvious is the time consumption, we estimated a time saving from ca. 30\%, for NMR spectra with less than 5 signals, up to $70 \%$ for more complicated NMR spectra. Other advantages include the possibility: to analyze various types of NMR nuclei such as $14 \mathrm{~N}, 19 \mathrm{~F}$, and 31P; to write reports without the need of NMR software but only a copy of the NMR spectrum, the chemical shift values and access to Microsoft Excel software; to avoid typos and format mismatches; to quickly correct the spectrum interpretation and instantly generate a new output; to avoid manual calculations of the coupling constants. From the educational point of view, the use of ASpin leaves to the users the full possibility to interpret the spectrum, in this way users can gain more experience in the interpretation process, without giving up the time saving. Nevertheless, ASpin doesn't require any license and it is free of charge.

Nowadays, NMR facilities are present in any department of chemistry/biology in the world, any chemistry related research institute and many chemical industries. Any undergraduate, postgraduate, researcher, technician involved in synthetic chemistry is a potential NMR user. In this scenario, ASpin could become a widely used user-friendly tool not only for academia.

\subsection{The process}

In Figure 1 is reported an example of $1 \mathrm{H}$ NMR spectrum for a generic organic molecule:

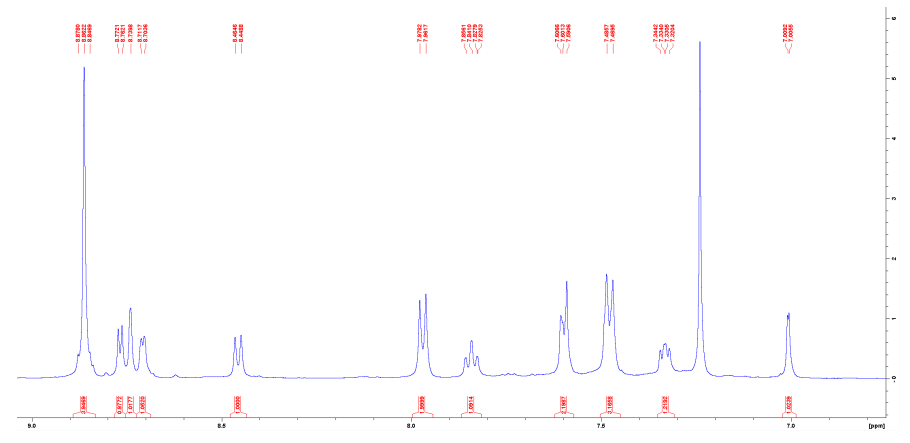

Figure 1. Expansion of $1 \mathrm{H}$ NMR spectrum for a generic molecule, including integration (bottom red values) and chemical shift (top red values).

The spectrum has to be reported in accordance with the publisher guidelines but generally it is templated as follow: ${ }^{1} \mathrm{HNMR}(500 \mathrm{MHz}, \mathrm{CDCl}) \delta$ : $8.86(\mathrm{~s}, 4 \mathrm{H}) ; 8.77(\mathrm{~d}, \mathrm{~J}=5.00 \mathrm{~Hz}, 1 \mathrm{H}) ; 8.73(\mathrm{~s}, 1 \mathrm{H}) ; 8.71(d, J=$ $4.05 \mathrm{~Hz}, 1 \mathrm{H}) ; 8.46(d, J=7.90 \mathrm{~Hz}, 1 \mathrm{H}) ; 7.97(d, J=8.25 \mathrm{~Hz}, 2 \mathrm{H}) ; 7.84(t, J=7.55 \mathrm{~Hz}, 1 \mathrm{H})$ Taking a closer look at the NMR report and the spectrum reported above, in Figure 2 are depicted all the graphic elements to transcript and the detailed component of the report. 


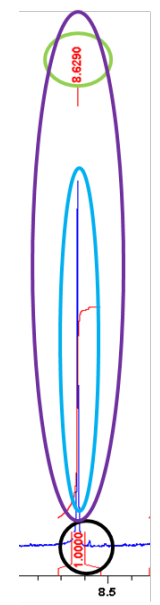

The NMR report includes:

- The isotope

- The NMR spectrometer frequency

- The used solvent

- The chemical shift

- The multiplicity

- The J coupling in $\mathrm{Hz}$ (to be calculated depending by the spectrometer frequency)

- The integration

Together with a specific punctuation sequence.

(1H) NMR $\left.400 \mathrm{MH}, \mathrm{CD}_{3} \mathrm{CN}\right) \mathrm{S} 8.95$ (d) $(2 \mathrm{H}) ; \ldots$

Figure 2. Elements included in the NMR report with corresponding graphic elements on the left and corresponding positions on the report header (bottom).

The report is generally generated manually by the user, who has to report the values from the spectrum in relationship with its "chemical" interpretation. This process can take from few minutes to up an hour. Any NMR dedicated software can generate a XY file indicating the chemical shift of the peaks inside a selected spectral region Figure 3.

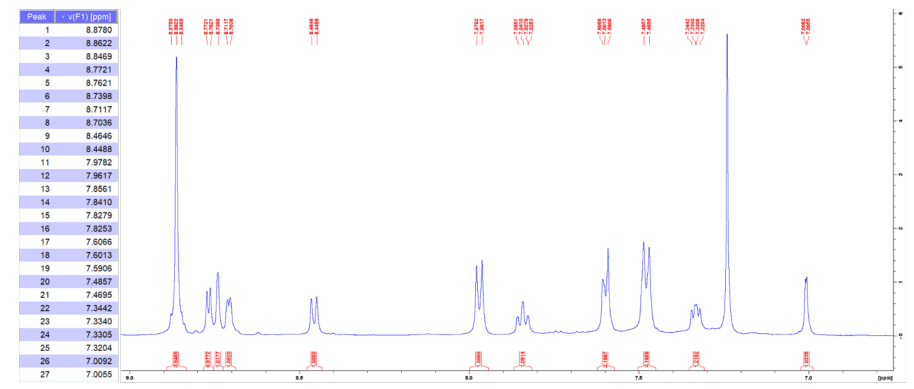

Figure 3. Column reporting the chemical shift values (left) of the selected spectral region (right), used software TopSpin by Bruker.

Generating the report with ASpin requires four easy steps:

1. The column reporting the shift (in decrescent order) for the desired spectrum has to be copied from the NMR software and pasted in cell B3 (first value) of the INPUT sheet of ASpin Figure 4.

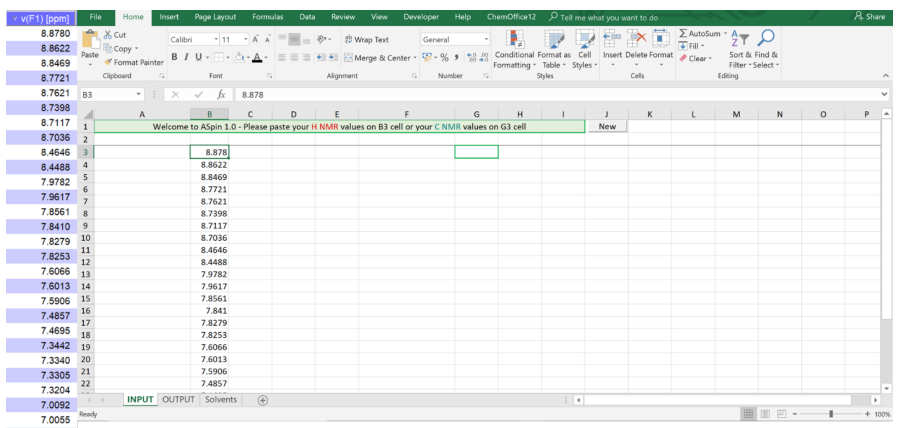

Figure 4. Peaks list and corresponding chemical shifts (left), ASpin INPUT sheet view (right).

2. The pasted values will appear on column A of the OUTPUT sheet (Figure 5). The user has to colour the cells accordingly with his/her interpretation of the multiplicity. The colour sequence is depicted in raw 3 and it corresponds to the standard colour in the "Fill color" button in the Excel "Home" toolbar. The cells have to be filled consecutively, except for singlet and multiplet, as follow: 
Dark red for singlet, one value only;

Red for doublet, two values;

Orange doublet of triplets, six values;

Yellow doublet of doublets, four values;

Light green triplet, three values;

Green triplet of doublet, six values;

Light blue multiplet, upper and lower values;

Blue quartet, four values;

Dark blue quartet $A B$, four values;

Purple pentet, five values;

Black sextet, six values.

Higher multiplicity can be treated as doublet or as triplet if they are even or odd numbers respectively, by selecting the corresponding central two (doublet) or three (triplet) values.The values in column A without a colour filling won't be processed and included in the report.

In cell $\mathrm{B} 4$ the spectrometer frequency (in $\mathrm{MHz}$ ) has to be indicated and in cell $\mathrm{B} 6$ are listed all the most common deuterated solvents. After colouring the desired values, picking spectrometer frequency and solvent, the user has to click on RUN button.

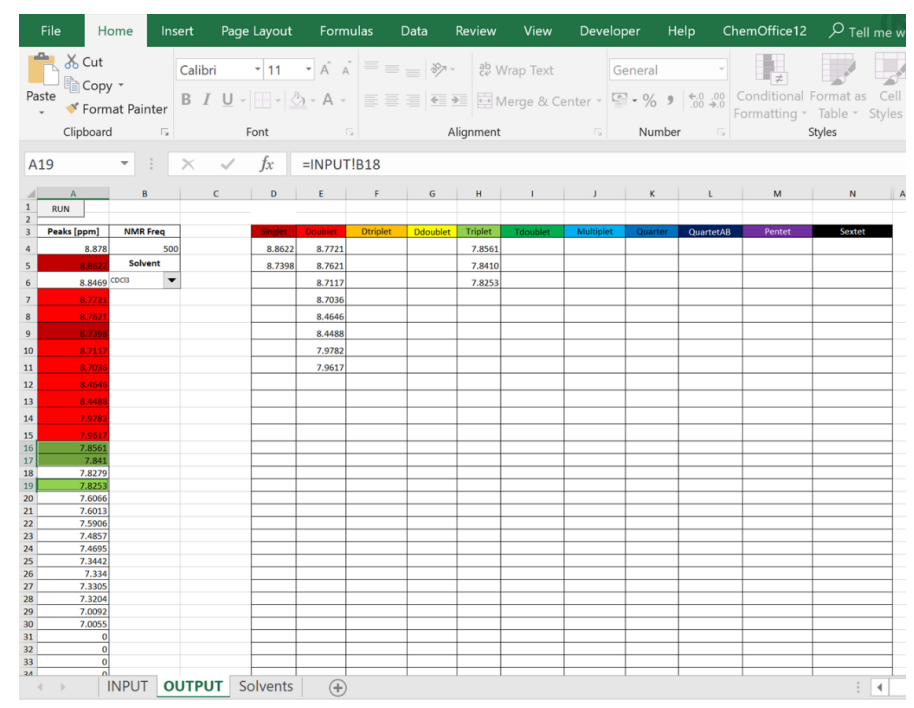

Figure 5. ASpin OUTPUT sheet view.

3. Depending by the assigned multiplicity, the J values will be automatically calculated. The signals list will appear in columns AQ-S, indicating multiplicity, chemical shift and coupling respectively. The user has to indicate the integration (AT) for each signal, followed by END (Figure 6). For a quick check, the total number of protons is indicated in cell AT45. 


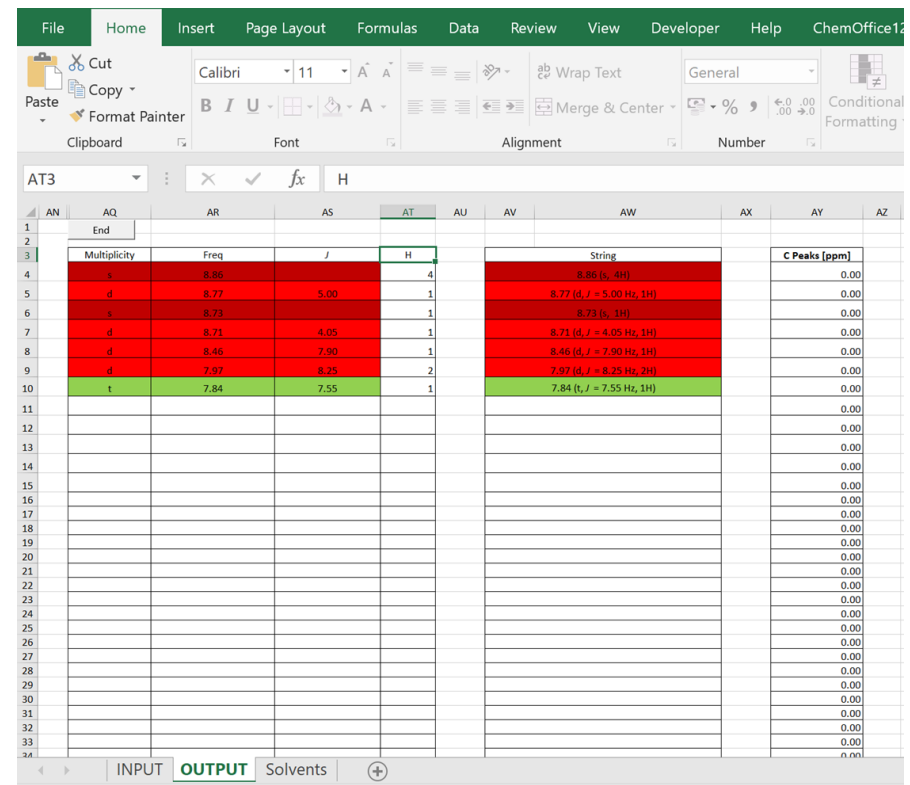

Figure 6. ASpin OUTPUT sheet view. In columns AV-AW, the individual signal characterization preview.

4. The desired NMR report will appear in a designated cell (BC7, Figure 7), ready to be copied and pasted in any manuscript.

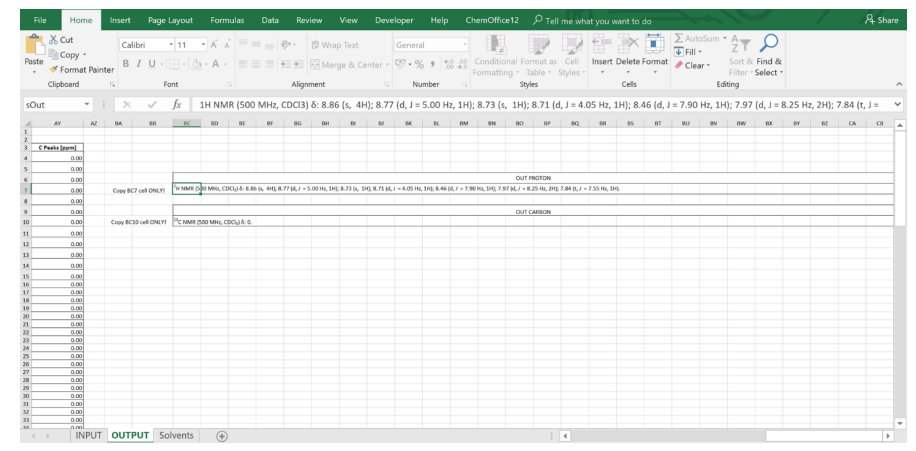

Figure 7. ASpin OUTPUT sheet view, in cell BC7 the final string containing the report.

For nuclei that do not require an interpretation but only to be listed (such as $13 \mathrm{C}$ and $31 \mathrm{P}$ ), the user has to repeat Step 1, pasting the column with shift values in column G in sheet INPUT. The values will the appear in column AY of sheet OUTPUT. By clicking the END button, the report will be generated in cell BC10.

To reset the INPUT sheet and run a new spectrum, the user has to click on "NEW", cell J1 of INPUT sheet.

Together with INPUT and OUTPUT, a third sheet named "Solvents" is included and it resumes the proton and carbon NMR shifts for the main deuterated solvents.

\section{Limits}

As any homemade software, ASpin is affected by some limits that perhaps will be enhanced in the future. The main limitations of this tool can be summarized as: only 100 values can be elaborated, only in decrescent order, leading to max 40 strings; in case of "broad" signals, the typical "b" or "br" before the multiplicity symbol has to be added manually; if the values belonging to the same proton/signal are mixed with the values of another proton/signal with the same multiplicity, these cannot be interpreted correctly by ASpin, to overcome this issue the values belonging to one proton/signal have to be swapped in the column B (INPUT) in order to avoid the crossing of the other signal with the same multiplicity; only one formatting is available to report the NMR characterization. 


\section{Future work}

The attached version of ASpin could be considered as a beta version. Future improvements will include a more user friendly graphic interface together with addressing possible feedbacks from the users or fixing unknown bugs.

Author Contributions: All authors contributed equally to the writing of this paper. All authors read and approved the final manuscript.

Conflicts of Interest: "The authors declare no conflict of interest."

(C) 2019 by the authors; licensee PSRP, Lahore, Pakistan. This report is an open access report distributed under the terms and conditions of the Creative Commons Attribution (CC-BY) license (http://creativecommons.org/licenses/by/4.0/). 